\title{
Promoting pediatric radiology at multiple levels
}

\author{
Mary L. Dinh ${ }^{1} \cdot$ Cory M. Pfeifer ${ }^{2}$ (I) $\cdot$ Ami Gokli $^{3}$ (D) \\ Received: 1 January 2021 / Revised: 8 March 2021 / Accepted: 15 April 2021 / Published online: 1 May 2021 \\ (C) The Author(s), under exclusive licence to Springer-Verlag GmbH Germany, part of Springer Nature 2021
}

Concerns have arisen over decreasing interest in pediatric radiology as a subspecialty despite increasing numbers of pediatric radiology jobs. For the 2019-2020 academic year there were approximately 140 unique new pediatric radiology job postings on the Society for Pediatric Radiology (SPR) website, an increase from approximately 100 in 2016-2017 [1]. Despite this, the number of pediatric radiology fellows has declined since participation in accredited pediatric radiology fellowships peaked in 2013 [2], with interest in pediatric radiology reaching a 6-year low in 2019 [3]. We discuss the trends in the pediatric radiology workforce and how they relate to interest in pediatric radiology fellowships [3]. We identify reasons for declining subspeciality interest and discuss potential solutions at the medical student, resident and fellow levels to increase the workforce.

Pediatric radiologists are in high demand in all types of practice settings because of the increased demand for subspecialty work [4]. The SPR and American College of Radiology (ACR) job boards demonstrate $50 \%$ and $60 \%$ increases in the number of available pediatric radiology jobs since 2017 [4]. Despite this high demand, pediatric radiology only represents about $5.5 \%$ of the radiology workforce [2].

Although there is an overall trend for radiology residents to subspecialize, pediatrics is the only subspecialty that showed consistently declining interest from 2013 to 2019 [3]. In 2017-2018 and 2018-2019, the number of graduating fellows, 55, was the lowest in 10 years, a stark comparison to the 92 fellows who graduated in 2013 [5]. At the completion of the 2019-2020 academic year, the number of pediatric fellows had only improved minimally at 58 , still a $27 \%$

Cory M. Pfeifer

Cory.Pfeifer@UTSouthwestern.edu

1 Penn State College of Medicine, Hershey, PA, USA

2 Department of Radiology, University of Texas Southwestern Medical Center, 5323 Harry Hines Blvd., Dallas, TX 75390, USA

3 Department of Radiology, Staten Island University Hospital, Staten Island, NY, USA decrease from 5 years prior [5]. As of October 2020, 21 accredited pediatric radiology programs had no fellows.

With such significant declining interest, the workforce is dwindling even as demand for jobs is surging. To compound this dwindling workforce, a large number of currently practicing pediatric radiologists are on the verge of retirement. A 2019 SPR survey estimates that $38 \%$ of current pediatric radiologists will retire in the next 10 years, further decreasing the workforce [6].

\section{Program director perspective: reasons for declining interest}

In 2020, the Radiology Research Alliance commissioned a survey of program directors that revealed that the most commonly perceived reasons for decreased interest in pediatric radiology are call responsibilities, a perception of lower compensation compared with other subspecialties, and inadequate exposure to the field (C.M. Pfeifer, personal communication, Dec. 1, 2020). Inadequate exposure could be related to scheduling because many residents do not have a sufficient pediatric rotation before the fellowship selection process. It might also be related to a lack of pediatric patients at smaller, nonpediatric hospital residency programs or a lack of funding to send residents to larger pediatric centers. Only $42 \%$ of community diagnostic radiology training programs include a rotation at a free-standing children's hospital [5], while that percentage increases to $55 \%$ for university and hybrid diagnostic radiology programs, with programs classified according to the FREIDA (Fellowship and Residency Electronic Interactive Database Access) residency program database [7].

When surveyed, residents have stated that they perceive job opportunities in pediatric radiology to be geographically confining, more limited in number, and lower in compensation [2]. Farmakis et al. [2] found that respondents indicated they would be more likely to consider pediatric radiology if there were more flexible job opportunities and higher demand for pediatric radiologists. 


\section{Program director perspective: addressing the issues}

There appears to be a disconnect between what residents perceive and the current atmosphere in pediatric radiology. This likely stems from miscommunication at many levels. At the medical student level, radiology fellows/residents might not have enough time to spend with students, and radiologists who do speak to medical students might not be focusing on the right groups of students. Finally, many medical schools do not have dedicated radiology rotations and some require completion of a general radiology elective prior to offering a pediatric radiology elective. At the resident level, certain negative perceptions about pediatric radiology have been circulating, such as lower salary, heavy academic burden and too much call, although much of this is myth.

Call responsibilities vary hospital to hospital and region to region, and these duties are not generalizable. Pediatric radiologists are distinct in that they perform life-saving imaging procedures such as intussusception reductions. Because of this skill, call responsibilities include being available for these procedures. At most institutions, the frequency of call is dictated by the number of pediatric radiologists in the call pool. At a standalone pediatric institution with 44 attending physicians, call is likely to be once a month for a late weekday shift and once a month for a weekend shift. At smaller institutions, call might be more frequent. Surveys completed by former fellows in pediatric radiology have shown that many take call from home and many institutions have nighthawk/teleradiology [6], and this might alleviate some of the extra call burdens that pediatric radiologists face from the effect of being in a small call pool. To gain a better perspective on call, future studies could address variations in call schedules and how to level the playing field.

Salary also depends on many factors [8]. Accurate salary reporting in all of radiology is not widely available because $75 \%$ of radiologists are employed by private practices and are not required to report salaries. Just like all other specialties, salaries depend on many factors including geography (different tax situations and retirement options) and relative value units (RVUs). In terms of geography, average salaries for general radiologists are highest in the Southeast at $\$ 425,000$, while in the Northeast average salary is $\$ 310,000$ and in the West, $\$ 300,000$ [8].

Nationwide U.S. data from pediatric radiologists at private and academic hospitals show that the 50th percentile total compensation for all participants is $\$ 390,000$ on average; the nationwide average for general radiologists is $\$ 345,000$ [8]. Additionally, results from a survey of recently graduated pediatric radiology fellows showed that $40 \%$ of fellows began with an initial salary greater than $\$ 400,000$ in 2019 , an increase of $12 \%$ from 2017 [6].
In $2015,30 \%$ of all pediatric radiology fellows were concentrated in three programs located in Boston, Philadelphia and Cincinnati [2]. As of October 2020, this percentage increased to $43 \%$ [9]. Residents might believe they need to choose one of these programs, but the lack of geographic diversity could be a travel burden on residents who hail from other areas in the United States and would be forced to move from friends and family for the duration of their fellowship. Furthermore, concentrating fellows into a few large training centers can have downwind effects on smaller training programs. For example, having fewer or no fellows to train might mean that faculty are less attracted to these smaller programs.

\section{Program director perspective: opportunities}

There is opportunity to increase interest in pediatric radiology at the medical student, resident and fellow levels. Medical students should be engaged with early and often. Attention should be paid to medical students interested in radiology as well as students interested in pediatrics [10]. Pediatric-interested medical students might become more interested in radiology if they understood how central radiologists are in clinical care and patient management, and our role in patient contact, hands-on US and pediatric radiology procedures. Rather than a separate month-long pediatric radiology elective, general radiology electives should include time at the closest children's hospital to increase exposure, research opportunities and mentorship within the field. Giving medical student lectures and speaking at interest group meetings can be key to increasing exposure. At the resident level, it is essential to introduce pediatric radiology early in residents' training through lectures and mentorship. It is also important to address and dispel myths about low salary, research expectations, etc. At the fellow level, we must encourage residents to accept fellowship positions at diverse pediatric radiology institutions. A vast number of programs encompass an excellent breadth of pathology. Program directors from all pediatric radiology programs should encourage their applicants to interview at a number of programs before deciding. Residency program directors should consider allowing a larger number of interview days so residents can be exposed to more programs.

\section{Program director perspective: current fellowship situation}

The pediatric radiology fellowship process is not part of the National Resident Matching Program (NRMP) at this time. Because there is a large surplus of positions compared to the number of applicants, and so many applicants choose one of the three fellowship programs that have up to 10 positions per year, the smaller programs struggle to fill the few open spots they have. To remain competitive, programs offer earlier and 
earlier interview dates. This creep up of interview start dates forces residents to apply earlier and make earlier decisions, depriving them of time and exposure to some fields [11]. This is a well-known problem in all non-NRMP fellowship application processes. The Society of Chairs of Academic Radiology Departments (SCARD) recently created a "handshake" policy for a universal interview start date for subspecialties not participating in the match. This policy is likely to decrease interview cancellations and remains in effect for the 2020-2021 academic year.

The Radiology Research Alliance survey for 2020 confirmed that most program directors in pediatric radiology report fewer fellows interviewed in recent years [12]. All of the program directors who responded reported adhering to the new interview start date policy. Although $63 \%$ of program directors support a universal timeline for fellowship applications, when asked about the possibility of a universal match, most were against the idea, with $32 \%$ in support and $32 \%$ undecided. In the authors' opinion, a universal start date for fellowship applications is a positive step because it will help residents not feel rushed into a decision.

\section{Moving forward}

Opportunities to increase interest in pediatric radiology include increasing exposure, dispelling myths, encouraging diverse fellowship choices, and engaging with medical students earlier in their education. Fellowship interviews were virtual in 2020-2021 because of the coronavirus disease 2019 (COVID-19) pandemic. Program directors are doing their part to help with fellow recruiting by agreeing to the universal interview start date. To help with pediatric radiology recruitment, a committee led by Dr. Shannon Farmakis has been formed through the American College of Radiology (ACR) and will focus on addressing workforce shortages through education and collaborative relationships. The committee has recently collaborated with the SPR and created a video about dispelling myths in pediatric radiology that is publicly available on YouTube and has been distributed through the SPR listserv. The committee is devoted to reaching medical students/residents early in their education to show them the rewards of a career in pediatric radiology.

\section{Declarations}

Conflicts of interest None

\section{References}

1. Pfeifer CM (2019) A comparison of neuroradiology and pediatric radiology job boards. Curr Probl Diagn Radiol 48:329-332

2. Farmakis SG, Hardy AK, Thomas KB et al (2019) Changes in factors influencing fellowship choices among radiology residents from 2008 to 2018 and methods that may increase interest in the pediatric radiology subspecialty. Pediatr Radiol 49:1132-1141

3. Ballard DH, Summers D, Hoegger MJ et al (2020) Results of the 2019 survey of the American Alliance of Academic Chief Residents in Radiology. Acad Radiol. https://doi.org/10.1016/j. acra.2020.04.042

4. Pfeifer CM (2020) Lessons from the ACR and SPR job boards, 2016-2020. Presented at SPR education: existential threats and opportunities in pediatric radiology, 2020. Society for Pediatric Radiology website. https://myedu.pedrad.org/p/Existential. Accessed 5 Apr 2020

5. Accreditation Council for Graduate Medical Education (2019) ACGME data resource book. https://www.acgme.org/About-Us/ Publications-and-Resources/Graduate-Medical-Education-DataResource-Book/Graduate MedicalEducation/ GraduateMedicalEducationDataResourceBook. Accessed 12 Nov 2020

6. Society for Pediatric Radiology (2021) Physician resources committee. SPR website. https://www.pedrad.org/About-SPR/ Committees/Physician-Resources. Accessed 12 Nov 2020

7. Dinh M, Gokli A, Pfeifer CM (2021) Children's hospital experiences: do timing and prevalence of rotations contribute to the shortage of pediatric radiologists? Presented at the 2021 annual meeting of the American Roentgen Ray Society, April 18-22, 2021, virtual meeting. https://apps.arrs.org/eposters21. Accessed 14 Apr 2021

8. Hawkins M (2019) 2019 review of physician and advanced practitioner recruiting incentives. https://www.merritthawkins.com/ uploadedFiles/MerrittHawkins_2019_Incentive_Review.pdf. Accessed 5 Apr 2021

9. Accreditation Council for Graduate Medical Education (2021) Advanced program search database. https://apps.acgme.org/ads/ Public/Programs/Search. Accessed 5 Dec 2020

10. Bloom DA (2020) The future is now: insights into finding tomorrow's pediatric radiologists. Pediatr Radiol 50:161-163

11. Gokli A (2020) Pediatric radiology fellowship recruiting update. Presented at SPR Education: Existential Threats and Opportunities in Pediatric Radiology, 2020. Society for Pediatric Radiology website. https://myedu.pedrad.org/p/Existential. Accessed 5 Apr 2020

12. Pfeifer CM, Reddy R, Burton KR et al (2021) The evolving status of fellowships and mini-fellowships in diagnostic radiology: a survey of program directors and chief residents. Acad Radiol. https:// doi.org/10.1016/j.acra.2021.03.007

Publisher's note Springer Nature remains neutral with regard to jurisdictional claims in published maps and institutional affiliations. 